\title{
XVII. Observations and experiments upon Dr. James's powder, with a method of preparing, in the humid way, a similar substance
}

\author{
Richard Chenevix Esq. F.R.S. M.R.I.A.
}

To cite this article: Richard Chenevix Esq. F.R.S. M.R.I.A. (1801) XVII. Observations and experiments upon Dr. James's powder, with a method of preparing, in the humid way, a similar substance, Philosophical Magazine Series 1, 11:42, 108-112, DOI: 10.1080/14786440108675964

To link to this article: http://dx.doi.org/10.1080/14786440108675964

曲 Published online: 18 May 2009.

Submit your article to this journal

Џ Article views: 2

View related articles $\sqsubset$ 


\section{$[108]$}

XVII. Obfervations and Experiments upon Dr. JAMEs's Powder, suitb a Metbod of preparing, in the bumid Way, a fimilar Subfance. By RichaRd Chenevix, Efq. F.R.S. M.R. I. A.*

A

FTER the obfervations and experiments made by Dodor Pearfon, to inveftigate the nature of Doctor James's powder, and prefented by him to this fociety, very little remained to be effeeted or defired towards a further knowledge of the fubjeet. But thofe very experiments ferved to fuggef, that the mode of preparation was far from being the beft that the prefent improved ftate of chemical knowledge might afford; and that, in all probability, a lefs defective compofition might refult from a procefs more conformable to fome improvements, which of late have been advantageoufly applied to pharmaceutic chemiftry.

It may be laid down as a general principle, that, in delicate experiments, whether analytical or fynthetical, fire (that potent and once believed to be univerfal agent) is too precarious in its means, and too uncertain in its application, to be employed with full and conftant fuccefs. And, if it is ftill recurred to, the advantage of promptnefs, and a remnant of antient cuftom, are the principal reafons. But, where other methods can be devifed to effeet the fame combinations, (and the humid way offers many,) every perfon converfant in chemical knowledge will allow the benefit of adopting them, The recent improvement in the mode of preparing calomel, is a ftriking example of fuch falutary corrections being fuccefsfully introduced.

A few obfervations upon the formula according to which Dr. James's powder, or the pulvis antimonialis, is prepared, and upon forne properties of antimony, will place this affertion in a more prominent point of view.

In order to prepare this powder, we are told to take equal weights of bone or harthorn fhavings and crude antimony, and calcine them together, at a high temperature: in other words, to take phofphate of lime, which already contains a great excefs of lime, and add to it an oxide of antimony. In this procefs, it has been fuppofed, that the phofphoric acid of the bone or hartfhorn will faturate, not only the lime with which it was originally combined, but, in addition to it, a new portion of metallic oxide, and a new portion of lime. For, what little fulphuric acid might, during the procefs,

- From the Pbilojophical Tranjactions for s $80 \mathrm{x}$. 
have been formed by the combuttion of the fulphur of the crude antimony, is diffipated at a much lower temperature than that to which the powder is expofed.

Every oxide of antimony with which we are acquainted is volatile at a high degree of heat: it would therefore be hazardous to affert, that it is poffible to preferve always the fame proportion of antimony, whatever care may be employed in directing the operation; and a diffimilarity in the chemical refult muft neceffarily be attended with uncertainty in the medical application.

To this property may be added another, no lefs conducive to error. That portion of oxide of antimony which is not volatilized, becomes, in a great meafure, infoluble in all the acids. What the effeet of the gaftric juice may be upon a fubftance which refifts the action even of nitro-muriatic acid, it is not my purpofe to determine. It is fufficient for me to fay, that, as the quantity of infoluble matter in a given quantity of Dr. James's powder, prepared at different times, may vary, the effect of any dofe alfo may differ according to the proportions of foluble and infoluble matter.

I look upon it as a fortunate circumftance, that thofe experiments and obfervations which I mentioned in the beginning of this paper, exifted as a ftandard to which I might refer my own attempts, and by which I might eftimate their validity. Dr. Pearfon has proved, (as by my own experiments I have found,) that in Dr. James's powder about 28 per cent. refifted the action of every acid. In examining fome of the pulvis antimonialis of the London pharmacopoeia, I found the average quantity of infoluble matter to be about 44 per cent. This proportion, however, was liable to confiderable variation*.

The powder here treated of is denominated by Dr. Pearfon a triple falt, or a real ternary combination of a double bafis (lime and antimony,) with phofphoric acid. What I have mentioned with regard to the quantity of acid contained in bone or harthorn, as being too fmall to faturate a new portion of thefe bafes, may throw fome doubts upon the poffibility of any fuch combination in the prefent cafe. But 1 have made fome more direct experiments, which tend to prove that no fuch combination does exift.

I took fome white oxide of antimony, (formerly called

- I find, from the information of feveral medical gentlemen, that the pulvis antinzontialis is generally confidered as ftronger than Dr. James's powder. This feems rather extraordinary, when we confider that the quantity of infoluble matter is greater in the former than in the latter; and would almoft lead us to fufped it to be the active part of the medicine. 
algaroth powder,) precipitated by water from muriate of antimony, and beated it for a long time with phofphoric acid. I decanted the liquor, and wafhed the powder that remained. No antimony could be found in the liquor, nor could any traces of phofphoric acid be detected in the refiduary oxide of antimony. I then took a foltution of muriate of antimony, and divided it into two equal parts : into one I poured diftilled water; and into the other, $a$ folution of phofphate of foda. In each liquor, a copious precipitate was formed; which precipitates, after being well wafhed, were dried. The weight of both was the fame; whereas it is evident that, had any phofphoric acid been combined with the oxide, there would have been an augmentation of weight in that which was precipitated by the folution of phofphate of foda. This precipitate likewife, upon examination, gave no traces of phofphoric acid. From thefe experiments it appears that there exifts no combination which can be denominated a phofphate of antimony.

To attempt an explanation of the real nature of the powder here fpoken of, I had recourfe to fome experiments of M. Berthollet. By detonating fulphuret of antimony and nitrate of potafh in a crucible, he obtained a mals, which he reduced to powder, and wafhed. The liquor gave, upon evaporation, a cryftallized falt, which $M$. Berthollet terms an antimoniate of pota $\beta$. I never could fucced in any attempt to form a fimilar combination between the above white oxide of antimony and potan, owing, I believe, to the fmall quantity of oxygen contained therein, compared with that which is combined with the oxide obtained by detonation. I cannot therefore fay, that the powder in queftion is, in any degree, what M. Berthollet would call an antimoniate of lime.

But be the ftate, whether of mixture or of combination; what it may, my purpofe is to endeavour to produce a fubAance which, from its more certain mode of preparation, may be more equal and conftant in its effects.

Diffolve, together or feparately, in the leaft poffible portion of muriatic acid, equal parts of the forementioned white oxide of antimony and of phofphate of lime*. Pour this folu-

tion

* In order to procure the phofphate of lime, 1 diffolved, in muriatic acid, a quantity of calcined bone, and precipitated by ammonia, in its ftate of greateft caufticity. By this means, the excefs of muriatic acid, which held in folution the phofphate of lime, is faturated, and the phofphate is precipitated; but no muriate of lime is decompofed, if the ammonia is quite free from carbonic acid. This is the moft direet method of obtaining phofphate of lime pure. This falt is not decompofed, as tome havo afferted, by muriatic acid, but merely diffolved by it. I have been in* 
tion gradually into diftilled water, previoufly alkalizated by a fufficient quantity of ammonia. A white and abundant precipitate will take place, which, well wathed and dried, is the fubftitute I propofe for Dr. James's powder.

The theory of this precipitation is fo clear and fimple that it does not require any comment. It may be ufeful, however, to thofe who with to make this preparation, to remark, that it is abfolutely neceffary that the folution of phofphate of lime and of oxide of antimony, in muriatic acid, hould, after being well mixed, be poured into the alkaline liquor, in order to obtain a precipitate homogeneous throughout the operation. For, fhould the alkaline liquor be poured into the acid folution, the water of the former would act upon the entire mafs of oxide of antimony, while the alkali would precipitate the phofphate of lime only as it faturated the acid which held that falt in folution: thus the precipitate would contain more antimony in the beginning; and, towards the end, the phofphate of lime would be predominant. For the fame reafon, too, a pure alkali is preferable to its carbonate; for the carbonic acid difengaged would retain in folution a portion of phofphate of lime.

Whether this compofition be a chemical combination or a mixture, I will not take upon me to determine; but, for the reafons above mentioned, in fpeaking of Dr. James's powder, I belive it to be merely a very intimate mixture. At all events, it muft be more homogeneous than any that can be prepared in the dry way. 1t is entirely foluble in every acid that can diffolve either phofphate of lime or oxide of antimony feparately; and, to have it conftantly and uniformly the fame, no further addrefs in preparing it is required, than to avoid the errors I have mentioned.

As, after fome medical trials of the powder, it was fuggefted to me, that it might be advantageous to render it fomewhat ftronger, I prepared another portion, by taking two parts of oxide of antimony and but one of phofphate of lime, and precipitating as above defcribed. The medicinal power was then confiderably increafed.

Dr. James's powder is a medicine which has been fo long in ufe, and is fo defervedly ranked among the moft valuable we poffefs, that every attempt to render the procefs for preparing it more fimple and more certain, muft be allowed to be of

duced to ftate fully thefe particulars, becaufe, from the beneficial effects of this falt in the treatment of rachitis, as propofed by $\mathbf{M}$. Bonhomme, (Anrales de Cabimie, vol. xviii. p. Iry, ) it may become of general ule. The oxide of antimony, I obtained by precipitating, by water, the common butter of antimony of the fhops. 
fome importance. But whatever reafon there was to think, by arguing upon its chemical properties, that I had really fucceeded in improving its medicinal virtues, it ftill remained to be proved, by actual experiment, that the hoped-for fuccefs was not merely conjectural. To afcertain this, I gave fome of my powder to Dr. Crichton, Dr. Babington, and Mr. Abernethy; gentlemen whofe extenfive practice and acknowledged fkill fufficiently enabled them to judge of its medical properties. They all concur in opinion, that, in its general effects, it agrees with Dr. James's powder and the pulvis antimonialis; but that it is more mild, and confequently may be given in larger quantities, feldom producing naufea or vomiting in dofes of lefs than eight or ten grains.

XVIII. Obfervations and Experiments undertaken witb a Vierw to determine the Quantity of Sulpbur contained in Sulphuric Acid; and of this latter contained in Sulphates in general. By Richard CHBNevix, Efq. F.R.S. M.R.I.A.*

I

N a paper which I had the honour to prefent to the Royal Society of London, and the fubject of which was the analyfis of fome arfeniates of copper and of iron, I had occafion, in examining many pyrites, matrices of thofe ores, to remark the very great inequality which prevailed in the refults of repeated experiments, made with a view to determine the proportion of fulphur. But I foon perceived that the inaccuracy was caufed by a partial combuftion and acidification of the radical, through the means of the nitric acid employed to diffolve the ore.

Having therefore, in the ufual manner, afcertained what quantity of that ingredient remained untouched, I was forced to feek the reft of it in the liquor which had wathed the various precipitates. To obtain it, I poured a folution of nitrate of barytes into thofe wafhings when all the other fubftances had been carefully feparated, and was thereby enabled to precipitate, in a ftate of purity, the fulphate of barytes, formed by that earth, and by the portion of fulphur originally acidified in the firtt treatment of the ore by nitric acid. To come at the knowledge of the proportion of fulphur contained in a given quantity of fulphate of barytes, I had recourfe, in the firft inftance, to the quantity of fulphur faid by Lavoifier to be contained in fulphuric acid; and, in the

* From the Tranfactions of the Rojal Lrifh Academy. 